\title{
Encarnación, cognición y representación: ¿cómo piensan el cuerpo las ciencias cognitivas?*
}

\author{
Florent Coste \\ Traducción del francés al español de Rodrigo Zapata Cano \\ Universidad de Antioquia, Medellín, Colombia \\ rodrigozapatak@yahoo.com
}

\section{RESUMEN}

Las ciencias cognitivas intentan analizar el funcionamiento de la mente y el conocimiento. Constituyéndose en los años cuarenta y cincuenta del siglo XX, han utilizado el computador y la informática como modelos de referencia y han permitido desarrollar muchos proyectos tecnológicos (inteligencia artificial) y responder a múltiples desafíos epistemológicos. Pero desviadas, sin duda, por tan hechizantes perspectivas de su proyecto de conocer la cognición (memoria, lenguaje, consciencia, percepción, atención, dominio de la actividad motriz, etc.), y al caer en el olvido de la especificidad del ser humano, han sido conducidas a producir modelos de los que se excluían sobre todo la experiencia humana y el cuerpo.

Francisco Varela (1997) ofrece en el libro De cuerpo presente una síntesis de las actuales tendencias de la epistemología de las ciencias cognitivas con el fin de despejar las lagunas problemáticas, especialmente las que tienen que ver con la relación del cuerpo y la mente. En lugar de pensarlos separadamente, e inspirándose en Merleau-Ponty y el budismo, postula un "entrelazado" del cuerpo y el mundo, en el fondo del cual se realiza la cognición.

Palabras clave: ciencias cognitivas; cuerpo; mente; modelos cognitivos; fenomenología; budismo.

Las ciencias cognitivas han conocido dos desarrollos teóricos mayores. Francisco Varela intenta situarse e inscribirse en su prolongación, superándolos.

Cómo citar: Coste, F. (2020). Encarnación, cognición y representación: ¿cómo piensan el cuerpo las ciencias cognitivas? Ciencias Sociales y Educación, 9(18), 301-312. https://doi.org/10.22395/csye.v9n18a16

Traducción realizada por Rodrigo Zapata Cano del texto de Coste (2003).

Agradecemos a la revista Tracés. Revue de Sciences humaines por poner a disposición sus artículos para su publicación en otros idiomas. Nota del editor.

Recibido: 4 de mayo de 2020.

Aprobado: 10 de junio de 2020. 


\section{El cognitivismo}

La primera de estas se inspira en los trabajos de Jerry Fodor, alumno de Hilary Putnam. Fodor (1987)sostiene la hipótesis según la cual el "lenguaje del pensamiento" es una especie de lenguaje algebraico que convertiría las representaciones ordinarias en lenguaje abstracto, constituido por símbolos, sobre los cuales se efectuarían cálculos o cómputos. La cognición sería la manipulación de símbolos como en los computadores digitales. Pensar sería manipular símbolos y la cognición sería representación mental.

\section{El conexionismo}

El segundo modelo, el de la emergencia, consiste en asimilar el funcionamiento del cerebro a un modelo en red: muchas tareas cognitivas parecen efectuarse de manera óptima por sistemas que consisten en un gran número de componentes simples que, cuando están conectados según reglas apropiadas, producen un comportamiento global. Una operación cognitiva es el resultado emergente de pequeñas unidades interconectadas que interactúan entre sí, sin piloto central (se evacua el caso de la regresión del homúnculo). La representación consiste en la correspondencia entre un estado global emergente y las propiedades del mundo.

Así, en cada etapa, se encuentra planteada la pregunta: ¿cómo representa la cognición? Las ciencias cognitivas, reaccionando contra el behaviorismo que reducía la psicología al estudio de los comportamientos observables y no de estados mentales, han participado en la rehabilitación de la noción de representación. No queda por esto menos cargada de implicaciones epistemológicas. Para Jerry Fodor (1987), las representaciones se pueden identificar, como los hemos dicho, con símbolos de un "lenguaje del pensamiento". Así pues, los símbolos se definen por sus propiedades formales y últimamente por sus propiedades físicas. Según esto, el computador se comporta y efectúa sus programas de la misma manera que conoce o no el sentido de los símbolos: no los interpreta, se limita a manipularlos. El sistema solo interactúa con la forma de los símbolos y no con su sentido. Desde el punto de vista cognitivista, el cerebro se reduciría fácilmente a un simple aparato sintáctico y no semántico. Así, podría permanecer recluido en sus representaciones internas: Fodor (1987) y Chomsky formulan, además, la hipótesis de un "internalismo". Dicho de otro modo, el cognitivismo postula estados mentales que permanecen inaccesibles a la consciencia. No son inaccesible porque habiten esta región que se denomina "inconsciente", para luego ser llevada, por la reflexión o por un procedimiento regulado como el psicoanálisis, a la consciencia, sino que no estamos en condiciones de ser conscientes de estos procesos mentales regulados que dirigen nuestras representaciones mentales. De tal modo que el postulado fundamental del cognitivismo reside en 
el desfase, e incluso la zanja extendida entre el nivel personal y el subpersonal, más allá del nivel en el que las personas son verdaderas entidades.

Tres críticas mayores se le pueden dirigir a esta epistemología de la representación. En primer lugar, si sabemos que el programador participa en la elaboración semántica de los símbolos en un cómputo informático, se ignora perfectamente de dónde procede el contenido de sentido de las expresiones simbólicas, pues el cognitivismo medio supone su existencia en el centro de la consciencia. Así pues, es un origen misterioso de esta facultad de comportamiento inteligente para representar el mundo de cierta manera. En segundo lugar, el cognitivismo fragmenta al sujeto cognoscente: la cognición es cortada de la consciencia, que se relega a lo no esencial y solo mantiene relaciones con la representación. Por el contrario, ignora lo que nos es más familiar: nuestro sentido del yo que consiste en tener un punto de vista coherente y unificado, estable y constante a partir del cual pensamos, percibimos y actuamos. Por último, y como en la prolongación de este desconocimiento de la experiencia que postula que el cerebro es una especie de teatro interior de representaciones internas, desconoce totalmente nuestra interacción con el mundo y el medio externo que es susceptible de modificar el contenido de nuestros estados mentales. Hilary Putnam (2006), que vuelve sobre las teorías que había forjado, controvierte la posibilidad de darle un contenido simple a un concepto o a una noción: los objetos no existen independientemente de nuestros cuadros conceptuales. La significación es múltiple y se construye en la interacción. En síntesis, se le puede reprochar al cognitivismo olvidar nuestra consciencia, experiencia e inscripción en el mundo. Como diría Jackendoff (1998), "la mente computacional" se piensa contra "la mente fenomenológica".

De este modo, epistemológicamente, una teoría desarrollada de la representación subyace en el conjunto del discurso dominante de las ciencias cognitivas: 1) el mundo es predado, 2) nuestra cognición apunta sobre este mundo, 3) para tomar consciencia de este, nuestro trabajo consiste en representar estos vínculos y actuar luego sobre la base de estas representaciones. Como diría Rorty (2001), la mente todavía funciona como "un espejo de la naturaleza". De esta manera, la idea de un mundo o de un medio dotado de vínculos predados extrínsecos, que se ven reconstituidos por medio de un proceso de representación, se impone en el cognitivismo, donde persiste la afirmación fuerte de la concepción representacional de la mente inaugurada por Descartes. Jerry Fodor (1987) confiesa además que el cognitivismo es, desde su punto de vista, una avanzada mayor con relación al representacionismo de los siglos XVII y XVIII, en la única medida en que el computador se ha vuelto modelo de la mente. 
Por esta razón, el conexionismo intenta superar este movimiento y renuncia a hablar de representaciones en términos de símbolos, para analizarlos más bien en términos de valencias o vínculos. El conexionismo se deshace de los símbolos y responde al problema de la dirección no localizada de las informaciones en el cerebro. Así pues, propone un modo de funcionamiento del cerebro que es distribuido, cooperativo y autoorganizado ${ }^{1}$. Por ejemplo, Marvin Minsky (1986) estudia el sistema cognitivo como una sociedad y le confiere un lugar crucial a la arquitectura en patchwork [mosaico] de la cognición. La mente organiza microagentes susceptibles de resolver problemas localmente para formar "agencias" capaces de actuar a un nivel superior. De este modo, la mente emerge como una forma de sociedad. Pero, subsiste la metáfora tenaz de un agente cognitivo que ha caído en paracaídas en un mundo predado y que solo podría sobrevivir en posesión del mapa que lo describe.

No obstante, los enfoques en términos de emergencia o de sociedades de la mente hacen más problemática la noción de representación. ¿Qué hay de representacional en estas sociedades de la mente? Según Marvin Minsky (1986), la principal actividad del cerebro consiste, de hecho, en operar permanentemente modificaciones sobre sí mismo. Así, el cerebro produce recuerdos que cambian nuestras maneras de pensar ulteriores. A diferencia de una fábrica que produce automóviles, el cerebro produce y utiliza procesos capaces de modificarlo y modificarse ellos mismos. Se trataría de comprender los sistemas cognitivos, no sobre la base de su relación de entradas y salidas, sino por "su cierre operacional": los resultados de los procesos son procesos en sí mismos. Las clases de procesos en su funcionamiento se vuelven hacia ellos mismos para formar redes autónomas. En vez de representar un mundo independiente, hacen emerger un mundo como campo de distinción inseparable de las estructuras encarnadas en el sistema cognitivo. Así pues, se comienza seriamente a poner en duda la existencia en el interior del mundo de informaciones que el sistema cognitivo debería extraer.

\section{II}

En el libro De cuerpo presente, Francisco Varela (1997) toma otro punto de partida y trastorna la parcialidad de la perspectiva de un neuro-reduccionismo que nos limitaría a un "paquete de neuronas" (crick). De este modo, pretende rehabilitar la corporeidad de la inteligencia cognitiva: la vida de cada día implica agentes encarnados, es decir, listos para actuar, mientras que los principales elementos sensoriomotores están comprometidos en actividades paralelas. Encarnado,

El conexionismo ha ganado un renovado interés en el momento en que las matemáticas no-lineales han renovado sus problemáticas de autoorganización: podemos por ejemplo describir la coherencia adoptada por las propiedades emergentes gracias a la noción de "atractor" tomada de la teoría de los sistemas dinámicos. 
el hombre se encuentra siempre inmerso en una perspectiva. Para captar esta dimensión, algunas veces omitida por las ciencias cognitivas, el científico debe adaptar su discurso y privilegiar los relatos en primera persona y la naturaleza irreductible de la experiencia, rechazando la menor concepción dualista. Desde entonces, se opone a un internalismo a lo Chomsky o a lo Fodor que amurallaba la mente en sus representaciones mentales.

Inspirándose en la fenomenología, Francisco Varela adopta la perspectiva de Merleau-Ponty. La cultura científica nos invita a considerar nuestros cuerpos como estructuras a la vez físicas y vividas, exteriores e interiores, biológicas y fenomenológicas. Circulamos entre estos dos aspectos de nuestra corporeidad. Nos encontramos en un mundo, reflexionamos en un mundo que no es hecho, sino encontrado. Estamos en un mundo que parece haber estado allí, antes que la reflexión comience, pero este mundo no está, a pesar de esto, separado de nosotros. El reconocimiento de este círculo abre un espacio entre el yo y el mundo, revela un entredós, una vía media. Mi reflexión es reflexión sobre un irreflexivo. En tanto que tal, es acontecimiento específico y objeto de las ciencias cognitivas. Así pues, estas deben tener en cuenta esta ineludible reflexividad: a cada comportamiento le corresponde una estructura en el cerebro y, de manera recíproca, una modificación de esta estructura modifica mi comportamiento. Pero esta interdependencia descrita por el científico tiene lugar en el centro de su propia estructura cognitiva, la cual se encuentra en un horizonte de creencias y prácticas que interactúa con esta. Y postular este horizonte es también una actividad realizada por el ser vivo que soy, dotado de cierta corporeidad, inscrito aquí y ahora en una situación singular. Este conjunto de especificaciones recíprocas, que nos invita a volver incesantemente a nuestra particularidad, hace inadecuados los presupuestos de un observador desencarnado, como de un mundo que solo existe en la mente.

No obstante, la perspectiva fenomenológica tiene sus límites: al ofrecerse como una actividad posterior a los hechos, pierde así una gran parte de la riqueza de la experiencia. Husserl se propone estudiar la estructura de intencionalidad de la experiencia sin ninguna referencia al mundo factual y empírico. Sin embargo, este procedimiento de suspensión, que se ha denominado epojé, participa todavía de una especie de cartesianismo moderno que ignora el aspecto consensual y la dimensión corporal e inmediata de la experiencia. Así pues, Husserl se encuentra en el callejón sin salida de un movimiento puramente teórico (y no pragmático) hacia la experiencia: la fenomenología es a la vez interior al mundo (cualquier teoría presupone el mundo vivido) y exterior al mundo (solo la fenomenología puede volver a trazar el origen del mundo vivido en la consciencia). Nunca nos situamos fuera de la experiencia para hablar de esta. Para Merleau-Ponty (2010), perfectamente consciente de 
esta tarea circular, la fenomenología explica, desde luego, nuestra existencia concreta inscrita en un cuerpo, pero siempre volviendo después de los hechos: es una actividad teórica posterior a los hechos que no puede restituir de manera exhaustiva la riqueza de la experiencia.

¿Cómo ofrecer entonces un examen de la experiencia humana en sus aspectos a la vez reflexivos e inmediatamente vividos?

Varela (1997) propone entonces un nuevo modelo: la enacción ${ }^{2}$. Lejos de ser una representación de un mundo predado, una cognición es el acontecimiento conjunto de un mundo y una mente a partir de la historia de las diversas acciones que realiza un ser en el mundo. Esta perspectiva enactiva permite resolver algunos escollos del conexionismo y el cognitivismo y, sobre todo, su "ausencia total de sentido común", puesto que responde a la necesidad de ver en nuestras actividades los efectos de una estructura, sin por esto perder de vista la inmediatez de nuestra propia experiencia ${ }^{3}$. En efecto, la facultad de la cognición viva es plantear, en no importa qué momento, preguntas pertinentes que no están predefinidas, sino enactadas de manera contextual. De este modo, nuestro sentido común es requerido sin cesar para configurar nuestro mundo de objetos y se adapta a la flexibilidad de nuestros campos de observación: ningún aspecto de nuestro mundo natural y vivo se puede clasificar a partir de delimitaciones nítidas. Hilary Putnam (2006) dice incluso que somos incapaces de enumerar los objetos sobre la mesa (el lapicero, el borrador, el libro, pero ¿cuántas páginas en ese libro?). Propone así una alternativa a la noción muy caduca de representación. Se trata, a través de la enacción, para retomar una expresión de Jules Lequier (1936), de "hacer y, haciendo, hacerse": "Lejos de ser el conservatorio de significaciones fijadas y adquiridas, [la representación], haciéndose, expresa, por lo menos lateralmente, una ontogénesis de la cual hace parte" (Merleau-Ponty, 2010) ${ }^{4}$.

El mundo tal como lo percibimos no es independiente de aquel que lo percibe, sujeto y objeto, el que sabe y lo que sabe son la especificación recíproca y simultánea el uno del otro. A la manera de Merleau Ponty, que sobrepasa la simple oposición del realismo y el idealismo, Varela (1997) despeja una vía media: la enacción designaría una codeterminación de la gallina (el mundo implica reglas fijas y precede a la imagen proyectada en el sistema cognitivo) y el huevo (el sistema cognitivo crea su propio mundo). Las facultades cognitivas están inextricablemente vinculadas a la historia de lo que se ha vivido, se forjan por medio de la experiencia y no pueden eludir o evitar ese canal que es el cuerpo,

\footnotetext{
To enact significa suscitar, hacer que ocurra, hacer emerger.

3 Por ejemplo, Charles Taylor considera al ser humano como un "animal autointerpretante".

4 Originalmente a propósito del lenguaje. 
lugar de paso e interacciones del mundo y del sistema cognitivo. Desde entonces, la inteligencia que no funciona a partir de representaciones, ya no se define por su capacidad para resolver problemas, sino por la de penetrar un mundo compartido. El sistema cognitivo ya no formula soluciones y respuestas perentorias, sino que llega al mundo al mismo tiempo que hace ocurrir el mundo en la forma de la pregunta.

Así pues, está en la naturaleza de un sistema cognitivo funcionar en y gracias a una subjetividad encarnada. El cuerpo sería la cara visible de un entrelazado volteado y oculto de un proceso ontológicamente anterior, del cual es a la vez la limitación y la condición. Como estructura estabilizada, mediatiza un acontecimiento metafísico, imposible de captar directamente; solo es su indicio cristalizado en el cuadro de una ontología por esencia indirecta. En el cuerpo se voltea el primer movimiento de interiorización del mundo en una cara de síntesis supuesta del mundo. El organismo y el mundo se envuelven y descubren mutuamente en la circularidad fundamental que es la vida misma.

Según la enacción, el yo ya no es estable ni preconstituido. El mundo ya no es el cuadro de nuestra experiencia sobre el fondo del cual nuestro yo aparece como distinción y relieve. En síntesis, el mundo ya no es lo que se distingue del yo. Por consiguiente, el yo y el mundo ya no son dirigidos por una relación de distinción, sino por un engendramiento recíproco. De tal modo que la enacción participa en una reevaluación del cuerpo en las ciencias cognitivas. Lejos de ser un excedente embarazoso, la pura envoltura de una mente, el cuerpo se vuelve en efecto la condición de posibilidad de cualquier cognición, pues es fundamentalmente superficie metonímica donde lo común y lo privado se superponen. A partir de allí, el cuerpo funciona como un soporte cognitivo, lo hemos visto, pero constituye por consiguiente el medio de un anclaje más grande de las ciencias cognitivas en la experiencia humana y la base paradójica y ética en un mundo sin fundamentos.

\section{III}

Pues lo más pertinente del trabajo de Varela (1997) no reside en efecto en estas últimas consideraciones. No se contenta con exponer esta alternativa teórica a las fórmulas dominantes de las ciencias cognitivas. Hace un balance más filosófico que epistemológico del trabajo de las ciencias cognitivas. Como se sabe, tanto el cognitivismo como el conexionismo son llevados a formular ya sea teorías subpersonales o la hipótesis de una mente desprovista de un yo. En efecto, mientras que el cognitivismo cava una fosa entre la cognición como representación y la cognición como consciencia y lleva a pensar, aunque con reticencia, que "la consciencia no es buena para nada" (Jackendoff, 1998), el 
conexionismo, al considerar que el cerebro no es una entidad homogénea y unificada, sino más bien un conjunto heterogéneo de redes conectadas entre sí, diluye totalmente el yo. Constatando que la ciencia y la experiencia humana se dan la espalda una vez más, Minsky (1986) prefiere tomar la precaución de distinguir el "yo", que se refiere a una serie de acontecimientos mentales y corporales vinculados por cierta coherencia causal, y el "Yo" que remite a ese sentimiento que encuentra una esencia permanente donde actúa nuestra identidad. Por medio de esta pirueta terminológica mantiene así la existencia de lo que escapa a las ciencias cognitivas, dejándola en su margen y sin poder anexarla. Es como si las ciencias de la cognición se constituyeran en una carencia fundamental y se mantuvieran en esta laguna.

Varela (1997) saca dos conclusiones de este desconocimiento constitutivo de la experiencia humana:

-De una parte, estas fórmulas nos llevan a un estado de esquizofrenia y nos condenan a creer en algo de lo que sabemos (por nuestra constitución) que no existe. La ciencia nos conduce a negar el yo (fórmula de la mente despojada del yo), dejándonos en la incapacidad de renunciar a este impulso habitual hacia lo que acaba de sernos negado.

-De otra parte, Varela propone cuestionar esta no-coincidencia del distingo de Minsky como una incapacidad de las ciencias cognitivas para interrogar la base concreta de la representación que postulan y, mucho más, nuestra tendencia a aferrarnos a nuestro yo.

-Porque, detrás de estas formulaciones teóricas de una mente despojada de yo, permanece profundamente anclada la convicción de una unidad de la consciencia. Así pues, propone examinar este sentimiento que surge, desde que sentimos que ya no nos podemos fiar del yo o del mundo, como en un punto de referencia fijo y estable y que Richard Bernstein (2019) denomina la "angustia cartesiana". Formulémosla así: o nuestra consciencia posee un fundamento fijo o estable, de donde parte y descansa, o podemos escapar a una especie de confusión u oscuridad. Este sentimiento proviene de una necesidad enfermiza de un fundamento seguro que, si es insatisfecho, conduce al nihilismo: "El nihilismo radical es la convicción de una absoluta falta de solidaridad de la existencia, cuando se trata de valores superiores que se reconocen" (Nietzsche, 2000).

Y las ciencias cognitivas no escapan a esta tendencia nihilista, oscilando sin cesar entre mente y mundo, como en la búsqueda de un fundamento interior o exterior. Además, en una justa intuición de lo que es la enacción, Minsky (1986) hace en La sociedad de la mente el vínculo entre el nihilismo y la angustia cartesiana, y aborda nuestra incapacidad para encontrar un mundo plenamente 
independiente. El mundo no es un objeto, un acontecimiento ni un proceso. El mundo se presenta como un segundo plano, un campo que engloba el conjunto de nuestra experiencia, que no se deja captar fuera de nuestra estructura, de nuestro comportamiento y cognición. Lo que decimos a propósito del mundo dice por lo menos tanto sobre nosotros como sobre el mundo.

Desde entonces, ¿cómo escaparía la enacción a esta tendencia nihilista, la cual piensa en un engendramiento recíproco del mundo y del yo? En efecto, nuestra cognición emerge del segundo plano del mundo que se extiende más allá de nosotros, pero que no se puede captar fuera de nuestra corporeidad. De tal modo que no hay fundamento subjetivo (un yo estable) ni objetivo (el mundo es enactado al mismo tiempo que el yo). Además, cualquier búsqueda, ya sea de un fundamento exterior en el mundo o de uno interno a la mente, depende de esta oposición prefabricada objeto/sujeto, que Richard Rorty (2001) denomina "la invención de la mente como espejo de la naturaleza". Varela (1997) propone entonces un desarraigo del discurso de las ciencias cognitivas de la tradición occidental.

El libro De cuerpo presente vuelve (Varela, 1997) a insuflar el budismo en el discurso de las ciencias cognitivas como una tradición filosófica no abstracta y susceptible de anclar esta última en una experiencia humana. La casi totalidad de la vía búdica trata de los medios de superar el apego emocional del yo. Pone a disposición del lector dos tradiciones del budismo:

-En primer lugar, Varela (1997) propone adoptar la perspectiva que se deriva del método búdico del examen de la experiencia denominado "práctica de la atención". La doctrina búdica de la ausencia de un yo contribuye a comprender la fragmentación del yo descrita por el cognitivismo. La atención significa que la mente está presente en la experiencia cotidiana para sustraerla de la actitud abstracta: vivir lo que la mente hace cuando lo hace, coordinar cuerpo y mente (cualquier separación significa que no estamos presentes). El método de la atención se desarrolla en dos etapas: apaciguamiento de la mente (shamatha) y el desarrollo del discernimiento (vipashyana). Este método no nos obliga a aferrarnos a nuestro yo. La actitud abstracta que intenta erradicar no es solo la de la ciencia y la filosofía, sino también la de la vida cotidiana cuando el individuo no está atento (Varela habla de "escafandra" y "relleno de prejuicios y hábitos"). Lo que la atención perturba es la inatención, es decir, el hecho de estar distraídamente implicado sin darse cuenta. La atención es un ejercicio que los aprendices aplican en su propia respiración. Así pues, se desarrolla una perspectiva más panorámica, que es el descubrimiento de la consciencia. La atención se relaciona con las palabras aisladas de una frase. La consciencia es la gramática que engloba la frase. Experiencia del espacio y de las vastas 
dimensiones de la mente, la consciencia panorámica es el desarrollo natural de la meditación orientada hacia la atención. La reflexión ya no debe ser abstracta y desencarnada, sino encarnada, en la que el cuerpo y la mente se reúnen. La reflexión no solo apunta a la experiencia, sino que es una forma de la experiencia. Este método específico, que se puede comparar con el de un Hume incapaz de observar un yo, habría podido ser explotado par las ciencias cognitivas que teorizan una mente desprovista de yo con el objetivo de darse aplicaciones en lo cotidiano de la experiencia humana.

-En segundo lugar, abriéndose a la filosofía madhyamika de Nagarjuna ${ }^{5}$, un cierto no-dualismo, subraya Varela (1997), se puede yuxtaponer al entredós de Merleau Ponty y a la enacción, llevando hasta su conclusión lógica la noción de emergencia codependiente. Es una nueva tradición que permite superar el escollo del nihilismo y comprender que nuestro mundo no tiene fundamentos, pero también, y sobre todo, de aprender a vivir sin fundamentos. Putnam (2006) también hace la misma afirmación: "nuestra posición histórica (y no vemos su fin) es que debemos filosofar sin 'fundamentos'".

No solo cada momento de la experiencia toma la forma de una consciencia particular vinculada a un objeto particular de una manera particular, sino que todas las cosas están desprovistas de una naturaleza cualquiera, independiente e intrínseca, ya sea desde el punto de vista de los sujetos y objetos, las causas y efectos y cosas y atributos: "No se puede encontrar nada que no haya emergido de modo dependiente. Por lo tanto, nada se puede descubrir que esté desprovisto de fundamentos" (Kalapuhana, 1986) 6 . Nagarjuna (2018) introduce una diferencia considerable para la experiencia ampliando el abhidharma. Sin espíritu, mundo, polo subjetivo ni objetivo, no tenemos acto de conocimiento para realizar, puesto que no hay nada oculto y nuestra experiencia se produce siempre abierta y perfectamente revelada por lo que es. Así pues, la verdad es llamada relativa y convencional (samvrti), condición de posibilidad de algunas regularidades y conmensurabilidades en el mundo, como en los discursos cotidianos y científicos. Así, igual que la tradición madhyamika, en tanto que actividad provisional y convencional del mundo relativo que indica algo más allá de sí misma, del mismo modo la enacción debería designar más allá de sí misma una comprensión más verdadera de la ausencia de fundamentos. No obstante, no se trata de comprender esta ausencia como una negatividad. De manera general, el budismo acoge la ausencia de fundamentos como una gran bendición y abre el mundo como vía y lugar de realización. Nagarjuna escribe:

Madhyamika significa literalmente "vía media". Nagarjuna, inspirado por Buda, fue brahmán, especialista de los Vedas y de las ciencias hinduistas.

6 Cinco siglos después de Buda, durante la primera mitad del segundo siglo de la era cristiana, Nagarjuna es una figura mayor del budismo mahayana y vajrayana. Expone sus enseñanzas en forma dialéctica. 
"La verdad última no se puede enseñar separadamente de las prácticas cotidianas" (Nagarjuna, 2018).

El cuerpo se vuelve entonces propiamente indispensable para alcanzar la realización. Desde entonces, es el punto de articulación nodal de una formulación ética en ausencia de fundamentos. ¿Pero, cómo formular esta ética en tanto que el yo, portador de un poder moral, acaba de perder su consistencia? En la medida en que la sunyata es pérdida de fundamentos en el yo y en el otro como en su relación, el budismo, y en particular la tradición Mahayana, reinterpreta de hecho la ausencia de fundamentos (sunyata) como un desarrollo de la empatía, como un interés impregnado de benevolencia con respecto a los otros, una nonecesidad de la reacción del destinatario y una generosidad suprema. En efecto, el enfrentamiento de las propias tendencias con la avidez de la búsqueda de fundamentos es considerado un acto de amistad consigo mismo; a medida que se desarrolla ese sentimiento amigable hacia sí mismo, se amplía la preocupación con relación a su entorno. Pero esta empatía incondicional no depende de una conminación moral pragmática ni de un sistema ético axiomático, se define en respuesta a una situación particular y no sigue reglas. En el caso en que la preocupación empática sea engendrada en el contexto de atención/vigilancia, podemos decir que este saber-hacer está basado en la capacidad de respuesta hacia sí mismo y hacia los otros, en tanto que seres sensibles que sufren porque se aferran a un yo del cual, de hecho, están desprovistos. Por consiguiente, la ausencia de fundamentos puede revelarse, en un plano ético, como empatía no-egocéntrica, como una solicitud englobante y descentrada. Al final, desde su discurso de naturaleza epistemológica sobre las ciencias cognitivas, Varela (1997) logra evitar una reinterpretación de la condición posmoderna como una ética de las raíces budistas de la empatía.

\section{IV}

Nuestra cultura científica y el pensamiento occidental en general no poseen tradición susceptible de implementar la cognición y la experiencia de manera directa y pragmática. En efecto, los enfoques pragmáticos y progresivos de la experiencia pueden transformar nuestra búsqueda del fundamento. Esta nueva pragmática se propone, de alguna manera, para transformar la experiencia humana de lo cotidiano y salir de la indecidible oscilación entre objetivismo y nihilismo. En el caso de las ciencias cognitivas, el desarrollo teórico lleva a alejarse de la experiencia humana y engendra una posición dividida que nos conduce a afirmar consecuencias difíciles de creer o aceptar. En resumen, una voluntad anima el proyecto de Francisco Varela (1997): darle a las ciencias cognitivas los medios de un impacto de sus teorías en la vida cotidiana. 
Por esta razón, se opera una constante rehabilitación del cuerpo y de la particularidad de la encarnación en Varela (1997), quien es llevado a reformular los problemas más importantes de las ciencias cognitivas: ¿qué podría ser una red neuronal, para poder sostener un dasein, una existencia encarnada? Así pues, el cuerpo debe captarse simultáneamente como estructura experiencial y como medio de los mecanismos cognitivos, allí donde Merleau Ponty nunca dejó de volver. La actividad operante del cuerpo procede de un dato original que regresa en potencia activa de ser al mundo.

\section{Referencias}

Bernstein, R. (2019). Más allá del objetivismo y el relativismo. Ciencia, hermenéutica y praxis. Prometeo.

Fodor, J. (1987). El lenguaje del pensamiento. Alianza.

Jackendoff, R. (1998). La consciencia y la mente computacional. A. Machado Libros S. A.

Kalapuhana, D. (1986). Nagarjuna: The Philosophy of the Middle Way. State University of New York Press.

Lequier, J. (1936). La Liberté. Vrin.

Merleau-Ponty, M. (2010). Lo visible y lo invisible. Nueva Visión.

Minsky, M. (1986). La sociedad de la mente. Galápago.

Nagarjuna (2018). Fundamentos de la vía media. Alianza.

Nietzsche, F. (2000). La voluntad de poder. Edaf.

Putnam, H. (2006). Razón, verdad e historia. Tecnos.

Rorty, R. (2001). La filosofía y el espejo de la naturaleza. Cátedra.

Varela, F. (1997). De cuerpo presente. Gedisa. 